\section{Sub-cellular Distribution of the Substance $P$ in the Nervous Tissues}

Hebb and Whittaker ${ }^{1}$ have described the isolation of a sub-cellular fraction of brain tissue which contains bound acetylcholine and choline acetylase. This fraction was examined further by Whittaker ${ }^{2}$ with equilibrium centrifugation in a density gradient. His results appear to provide evidence for physiological significance of his "synaptic vesicle fraction". We have also attempted to apply his fractionation method to study sub-cellular distribution of substance $P$ in the nervous system.

The preparation and fractionation of homogenates of brain and spinal cord (rabbit or guinea pig) were carried out as described by Whittaker, the microsomal and the cytoplasmic fraction not being separated but combined as the supernatant fraction. Eserine was not used except for experiments on acetylcholine. Assay of substance $P$ activity was carried out on the isolated guinea pig ileum in the presence of atropine, pyribenzamine and tryptamine, its specificity being usually ascertained by desensitization treatment of the iloum strip with a large amount of substance $P$ prepared from the bovino brain by Euler's method ${ }^{3}$. Assay of acetylcholine was carried out on frog's rectus abdominis muscle.

The highest activity of substance $P$ (per gm. wet tissue and per gm. protein estimated by Lowry's method $^{4}$ ) was found in the synaptic vesicle fraction and then in the supernatant fraction, while the mitochondrial and nuclear fraction contained only a small amount of substance $P$. Such a sub-cellular distribution of substance $P$ was observed on all samples prepared from different regions of the central nervous system of rabbit and guinea pig; cortex, midbrain. cerebellum, ventral and dorsal halves of spinal cord. Of course, the total substance $P$ activity recovered was higher in the midbrain and dorsal spinal cord, while homogenates of cerebellum and ventral cord were lowest in its activity as reported by previous workers ${ }^{5,6}$. When the sub-cellular distribution of substance $P$ and acetylcholine in the spinal cord of rabbit was compared, it was found, as shown in Fig. 1, that the distribution of substance $P$ might be said to be nearly identical with that of acetylcholine, if the word 'ventral' for acetylcholine is replaced with 'dorsal' and 'dorsal' with 'ventral'.

It seems worth noting here, however, that the activity of acetylcholine measured was the sum of free and kound form, while the treatment such as extraction by heating at $p H 4$ scarcely increased the activity of substance $P$ obtained; a fact which is similar to that found on the particulate 5-hydroxytryptamine by Whittaker ${ }^{2}$. The bound substance $P$
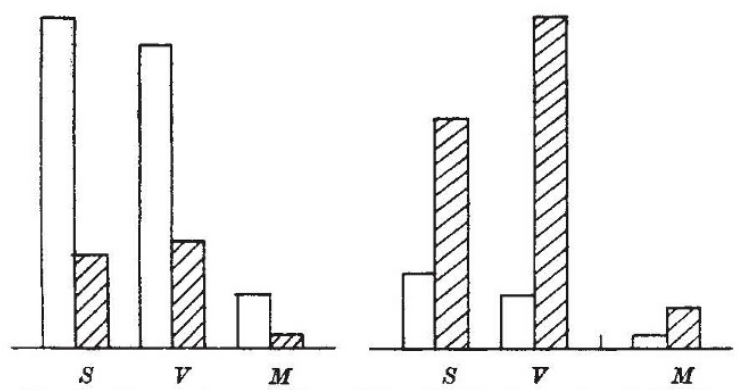

Fig. 1. Comparison of intracellular distribution of acetylcholine and substance $P$ in the spinal cord. Left, ventral half of the spinal cord; right, dorsal half of the spinal cord. Shaded, substance $P$; white, acetylcholine. $S$, Supernatant; $V$, vesicle

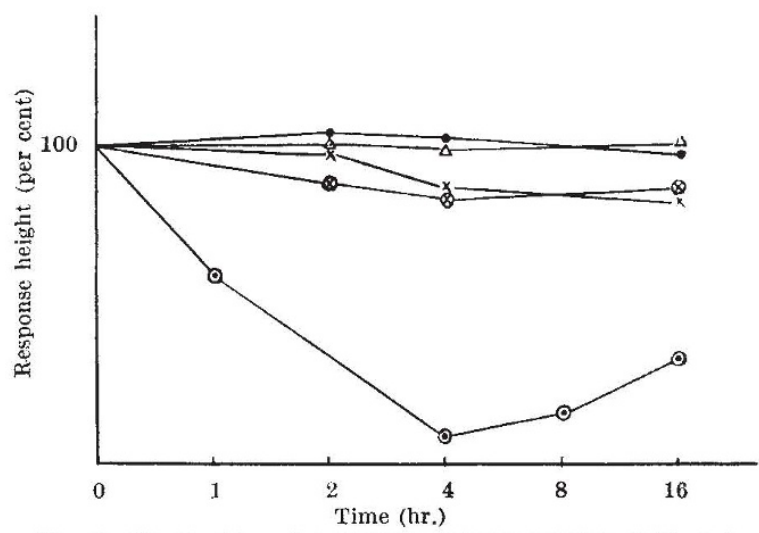

Fig. 2. Inactivation of substance $P$ by incubation with each fraction. $\odot$, Supernatant; $x$, vesicle; $\triangle$, mitochondrial; $\otimes$, fraction $A$;, nuclear. Fraction $A$ corresponds to Whittaker's $P_{\mathbf{8}} A$

may be released by treatment with sucrose solution. In this respect substance $P$ differs from acetylcholine and rather resembles 5-hydroxytryptamine.

It was also reported that choline-esterase activity was chiefly found in the fraction $S^{7,8}$. When each subcellular fraction was incubated with substance $P$, inactivation of the latter was observed only on incubation with the fraction $S$ (Fig. 2). Such an inactivation of substance $P$ was inhibited by adding strychnine $\left(10^{-3}\right)$ or lysergic acid diethylamine $\left(10^{-5}\right)$ into the incubation mixture, just as that of acetylcholine due to choline esterase by eserine. Lysergic acid diethylamine potentiates the gut-stimulating action of substance $P$. In this case, however, the response of ileum strip was too high to be ascribed to this potentiation effect alone, while strychnine $\left(10^{-3}\right)$ showed no potentiation of activity of substance $P$. Hence a specific enzyme or enzymes which inactivate substance $P$ seem to be present in the supernatant fraction ${ }^{9}$.

The increase in substance $P$ activity after incubation with the fraction $S$ for 5 or more $\mathrm{hr}$. was only an apparent ene, this gut-stimulating activity being not abolished by high dose of substance $P$. This component seems to be a kinin-like compound which causes a slow contraction of guinea pig ileum with a latency of considerable length and is more active on rat's uterus and colon than on the hen's rectum and not destroyed by trypsin ${ }^{10}$.

These results appear to suggest a close relationship of substance $P$ to the transmission mechanism in the central nervous system, even if it is not a transmitter itself.

Department of Physiology,

Akira Inouye KIYOSHI KATAOKA

Faculty of Medicine,

Kyoto University,

Kyoto, Japan.

${ }^{2}$ Hebb, C. O., and Whittaker, V. P., J. Physiol., 142, 187 (1958).

${ }^{3}$ Whittaker, V. P., Biochem. J., 72, 694 (1959).

${ }^{3}$ Euler, U. S. v., Acta Physiol. Scand., 4, 373 (1942).

${ }^{4}$ Lowry, O. H., Rosebrough, N. I., Farr, A. L., and Randall, R. J., J. Biol. Chem., 193, 265 (1951).

${ }^{5}$ Amin, A. H., Crawford, T. B. B., and Gaddum, J. H., J. Physiol., 126, 596 (1954).

- Pernow, B., Acta Physiol. Scand., 29, Supp., 105 (1953).

7 Toschi, G., Exp. Cell Res., 16, 232 (1959).

${ }^{8}$ Aldridge, W. N., and Johnson, A., Biochem. J., 73, 270 (1959).

'Tsujioka, T., J. Physiol. Soc. Jap., 20, 662 (1958).

${ }^{10}$ Krivoy, W. A., Brit. J. Pharmacol., 12, 361 (1957). "Inouye, A., Kataoka, K., and Tsujioka, T., Jap. J. Physiol.,
11,319 (1961). 